\title{
Simulation of the neutron response functions of diamond detectors with the NRESP code
}

\author{
Miroslav Zbořil*, Jorge Enrique Guerrero Araque, Ralf Nolte, Andreas Zimbal \\ Physikalisch-Technische Bundesanstalt, D-38116 Braunschweig, Germany \\ E-mails: Miroslav.Zboril@ptb. de, Jorge.Guerrero@ptb. de, \\ Ralf.Nolteeptb.de, Andreas.Zimbaleptb.de
}

\begin{abstract}
Detectors based on single crystal synthetic diamond are well suited for high resolution spectrometry of fast neutrons. Their radiation hardness and ability to operate in extreme conditions make them suitable for neutron plasma diagnostics for the ITER project. The task of plasma diagnostics via high resolution neutron spectrometry requires a good knowledge of the response matrix of the detector within a wide energy range. In this work, the Monte Carlo particle transport code NRESP is used for the calculation of the neutron response functions of diamond detectors in the energy range $7 \mathrm{MeV} \leq E_{\mathrm{n}} \leq 16 \mathrm{MeV}$. The NRESP code, originally developed at the PhysikalischTechnische Bundesanstalt (PTB) for the calculation of the neutron response functions of liquid scintillation detectors, was adapted for the simulation of the neutron-induced reactions which occur in a diamond detector. The simulated response functions are compared to measurements in quasi-mono-energetic neutron beams performed at the PTB ion accelerator facility (PIAF).
\end{abstract}

First EPS Conference on Plasma Diagnostics - 1st ECPD,

14-17 April 2015

Villa Mondragone, Frascati (Rome) Italy

\footnotetext{
*Speaker.
} 


\section{Introduction}

Radiation detectors based on synthetic single crystal Chemical Vapor Deposition (scCVD) diamond have shown a great potential for high resolution spectrometry of fast neutrons in harsh environments. In particular, their good energy resolution [1], fast signal response [2] and high radiation hardness [3] make diamond detectors suitable for neutron plasma diagnostics for the fusion project ITER. The task of plasma diagnostics via high resolution neutron spectrometry requires a good knowledge of the response matrix of the detector within a broad range of neutron energies $2 \mathrm{MeV} \leq E_{\mathrm{n}} \leq 20 \mathrm{MeV}$. Such a response matrix is needed for the unfolding of the measured pulse height spectra (PHS) in order to extract the full information about the incident neutrons.

As it is rather laborious to determine the complete response matrix experimentally, detailed simulations of the neutron response functions of diamond detectors are required. The simulation of a diamond detector response function is a difficult task as there is a number of neutron-induced nuclear reactions in carbon [4] which have to be taken into account. Standard neutron transport codes like MCNP are not well suited for this task as they cannot transport particles beyond the mass number of $\alpha$ particles. This is a severe limitation for the simulation of a diamond detector in which e.g. ${ }^{9} \mathrm{Be}$ ions are created in the reaction ${ }^{12} \mathrm{C}(\mathrm{n}, \alpha){ }^{9} \mathrm{Be}$ for neutrons of energies $E_{\mathrm{n}} \geq 7.2 \mathrm{MeV}$. Moreover, such neutron transport codes do not include the correlations between the individual reaction products with more than two particles in the exit channel, and the energy is conserved only in statistical limit. This is why in previous works $[5,6,7]$ the MCNP simulations were enhanced by additional ion transport subroutines and response functions of a diamond detector were simulated. These works were successful in describing well the sharp peaks in the diamond detector response functions but the simulations of wide distributions still suffered from the lack of detailed modelling of the individual reactions in carbon and reliable cross section data.

In this work, we describe our approach of modifying the Monte Carlo particle transport code NRESP [8] to adapt the products of the neutron-induced reactions in a diamond detector. With NRESP we have simulated nine PHS in the energy range $7 \mathrm{MeV} \leq E_{\mathrm{n}} \leq 16 \mathrm{MeV}$ and compared them with the experimental PHS obtained from measurements in mono-energetic neutron beams [9] performed at the PTB cyclotron. The diamond detector used in the experiment had dimensions of $4.6 \times 4.6 \times 0.5 \mathrm{~mm}^{3}$ and circular electrodes.

\section{Neutron spectrometry with diamond detectors}

In a diamond detector neutrons are detected via interactions with carbon nuclei. Depending on the incident energy $E_{\mathrm{n}}$, a fast neutron - we consider $2 \mathrm{MeV} \leq E_{\mathrm{n}} \leq 20 \mathrm{MeV}$ relevant for neutron plasma diagnostics in nuclear fusion - can undergo the following reactions $[4,8]$ :

1. Elastic scattering ${ }^{12} \mathrm{C}(\mathrm{n}, \mathrm{n}){ }^{12} \mathrm{C}$ is simply a neutron recoil on carbon nucleus.

2. Inelastic scattering ${ }^{12} \mathrm{C}\left(\mathrm{n}, \mathrm{n}^{\prime}\right){ }^{12} \mathrm{C}^{*}$, followed by a $\gamma$ ray emission from the excited state ${ }^{12} \mathrm{C}^{*}$. The energy threshold for this reaction amounts to $4.85 \mathrm{MeV}$.

3. Inelastic neutron-carbon interaction ${ }^{12} \mathrm{C}(\mathrm{n}, \alpha)^{9} \mathrm{Be}$, followed by emission of an $\alpha$ particle from the ground state of ${ }^{9} \mathrm{Be}$ (for $E_{\mathrm{n}}>7.2 \mathrm{MeV}$ ). 


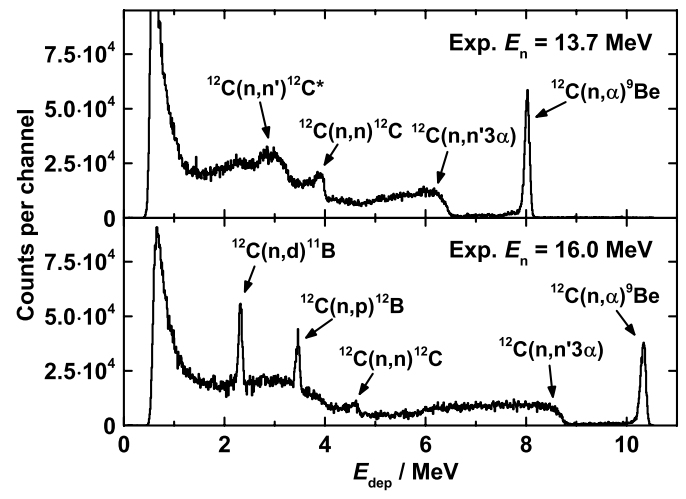

Figure 1: Two experimental PHS measured in mono-energetic neutron beams of $E_{\mathrm{n}}=13.7 \mathrm{MeV}$ and 16.0 MeV, respectively. The data are taken from [9].

4. For $E_{\mathrm{n}}>8.5 \mathrm{MeV}$ an inelastic neutron-carbon interaction ${ }^{12} \mathrm{C}\left(\mathrm{n}, \mathrm{n}^{\prime}\right)^{12} \mathrm{C}^{*}$ becomes important, followed by sequential emission of three $\alpha$ particles ${ }^{12} \mathrm{C}^{*} \rightarrow \alpha+{ }^{8} \mathrm{Be} \rightarrow 2 \alpha$. We denote this reaction as ${ }^{12} \mathrm{C}(\mathrm{n}, \mathrm{n} \prime 3 \alpha)$.

5. For $E_{\mathrm{n}}>8.7 \mathrm{MeV}$ another type of inelastic neutron-carbon interaction occurs, namely

${ }^{12} \mathrm{C}\left(\mathrm{n}, \alpha^{\prime}\right){ }^{9} \mathrm{Be}^{*}$ followed by emission of a neutron and two $\alpha$ particles, ${ }^{9} \mathrm{Be}^{*} \rightarrow \mathrm{n}+{ }^{8} \mathrm{Be} \rightarrow 2 \alpha$.

We denote this reaction as ${ }^{12} \mathrm{C}(\mathrm{n}, \alpha) \mathrm{n} 2 \alpha$.

6. For $E_{\mathrm{n}}>15.1 \mathrm{MeV}$ the reaction ${ }^{12} \mathrm{C}(\mathrm{n}, \mathrm{p}){ }^{12} \mathrm{~B}$ is followed by emission of a proton.

7. For $E_{\mathrm{n}}>15.8 \mathrm{MeV}$ the reaction ${ }^{12} \mathrm{C}(\mathrm{n}, \mathrm{d}){ }^{11} \mathrm{~B}$ is followed by emission of a deuteron.

Depending on the diamond crystal thickness, the charged products of the aforementioned reactions deposit a fraction of their energy inside the crystal. In general, sharp peaks in a measured PHS are observed only in case of reactions with charged particles in the exit channels (two-body reactions (3), (6) and (7)). The remaining reactions with a neutron in the exit channel give rise to wide distributions with characteristic edges. An example of two PHS measured in mono-energetic neutron beams of $E_{\mathrm{n}}=13.7 \mathrm{MeV}$ and $16.0 \mathrm{MeV}$, respectively, is shown in Fig. 1. It can be seen that the ${ }^{12} \mathrm{C}(\mathrm{n}, \mathrm{p}){ }^{12} \mathrm{~B}$ and ${ }^{12} \mathrm{C}(\mathrm{n}, \mathrm{d}){ }^{11} \mathrm{~B}$ peaks appear in the PHS for $E_{\mathrm{n}}=16.0 \mathrm{MeV}$ when the respective reaction thresholds are exceeded. The ${ }^{12} \mathrm{C}(\mathrm{n}, \alpha){ }^{9} \mathrm{Be}$ reaction results in a peak which is well separated from the smooth structure for $E_{\mathrm{n}}>9 \mathrm{MeV}$ and thus well suited for neutron spectrometry.

\section{Simulations with NRESP}

The Fortran-based code NRESP has been developed at the PTB since 1982 for the calculation of neutron response functions and detection efficiencies of liquid scintillation detectors [8]. In this code the neutron detection is simulated on an event-by-event basis. Partial spectra can be stored according to the first interaction of the incident neutron. The code contains cross section data for hydrogen, carbon and aluminium (detector housing) as well as a list of light output functions. Detailed modelling of the $3 \alpha$ breakup channels of ${ }^{12} \mathrm{C}$ is also included. In Fig. 2 the cross section data implemented in NRESP are plotted as a function of $E_{\mathrm{n}}$. The current modifications of the code can be summarized as follows: 


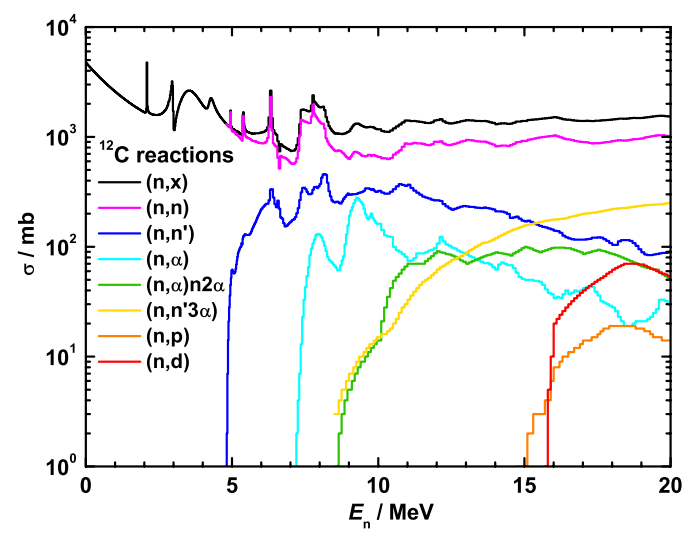

Figure 2: Cross sections of individual neutron-induced reactions on ${ }^{12} \mathrm{C}$ as used in the NRESP code. The black line marks the total cross section ${ }^{12} \mathrm{C}(\mathrm{n}, \mathrm{x})$.

- The hydrogen content was set to zero in order to simulate pure carbon (possible impurities, present in a diamond detector on the ppb level, were omitted). The geometry was set up in such a way that the aluminium housing, needed for scintillation detectors, can be neglected as well. The geometry is still limited to cylindrical shapes as in the original NRESP, but this does not represent a problem as the detector dimensions were set to match the sensitive volume of a real scCVD diamond detector.

- Cross sections for neutron-induced reactions in carbon were checked against the ENDF/BVII.1 library and a good agreement was found for all reactions shown in Fig. 2.

- NRESP uses non-isotropic angular distributions in the center-of-mass system for the reactions ${ }^{12} \mathrm{C}(\mathrm{n}, \mathrm{n}){ }^{12} \mathrm{C},{ }^{12} \mathrm{C}\left(\mathrm{n}, \mathrm{n}^{\prime}\right){ }^{12} \mathrm{C}^{*}$ and ${ }^{12} \mathrm{C}(\mathrm{n}, \alpha){ }^{9} \mathrm{Be}$. The angular distributions for the reaction ${ }^{12} \mathrm{C}(\mathrm{n}, \mathrm{n}){ }^{12} \mathrm{C}$, originally used in NRESP, were replaced by data taken from the ENDF/B-VII.1 library. Data for the ${ }^{12} \mathrm{C}(\mathrm{n}, \alpha){ }^{9} \mathrm{Be}$ reaction were taken from an evaluation of experimental PTB data [10].

- The ranges of $\alpha$ particles, protons and deuterons in carbon were calculated using the SRIM code [11] and implemented in the NRESP code in a tabulated form.

- The nonlinear light output function, used for description of scintillation detectors, was replaced by a linear relationship between the energy $E_{\text {dep }}$ deposited in the diamond detector and the resulting pulse height.

\section{Benchmark of simulations}

A set of nine experimental PHS was measured using quasi-mono-energetic neutron fields produced with deuteron beams from the PTB cyclotron incident on a deuterium gas target [9]. Monoenergetic neutrons from the $\mathrm{D}(\mathrm{d}, \mathrm{n})^{3} \mathrm{He}$ reaction were selected using the time-of-flight method. These PHS were used as a benchmark of the NRESP simulations. Figure 3 depicts the comparison between the simulated and measured PHS for nine cases of $E_{\mathrm{n}}$ ranging from $7.0 \mathrm{MeV}$ to $16.0 \mathrm{MeV}$. 

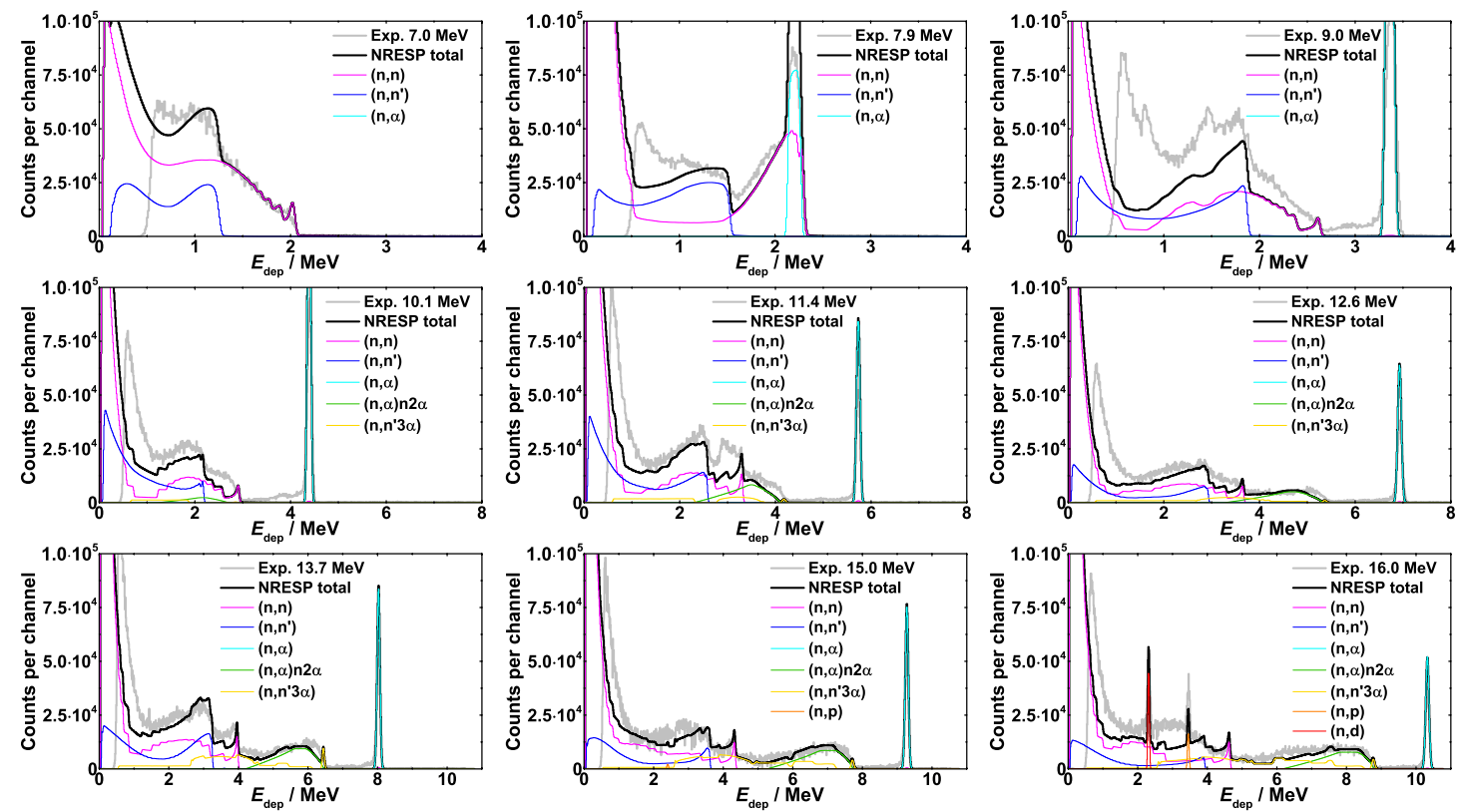

Figure 3: Comparison of the NRESP simulations to the experimental PHS measured in monoenergetic neutrons beams [9] at PIAF. The plots show the comparison at $E_{\mathrm{n}}=7.0,7.9,9.0,10.1$, $11.4,12.6,13.7,15.0$ and $16.0 \mathrm{MeV}$, respectively. In each plot the partial spectra of individual neutron-induced reactions are shown.

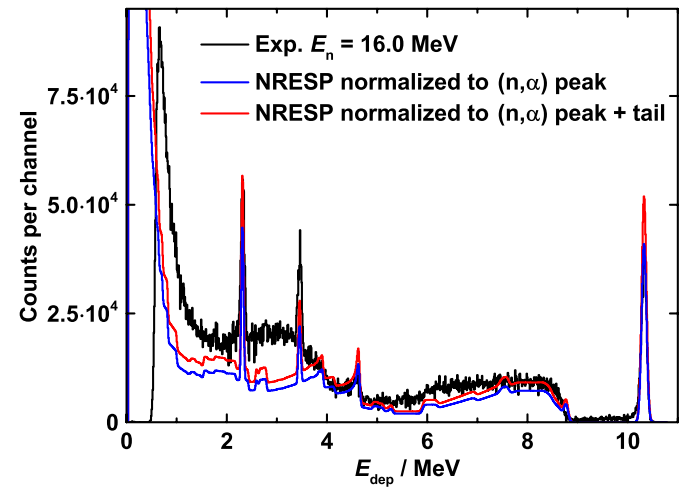

Figure 4: Comparison of two different normalizations of simulated PHS for $E_{\mathrm{n}}=16.0 \mathrm{MeV}$. The blue PHS was normalized to the area of the $(n, \alpha)$ peak in the experimental PHS, while the red PHS was normalized to the area of the same peak including its low energy tail down to $9 \mathrm{MeV}$ of deposited energy $E_{\text {dep }}$.

In each simulation the incident neutron energy $E_{\mathrm{n}}$ was set to the value known from experiment. In addition, the width of the neutron peak (energy spread) was represented by a uniform distribution around $E_{\mathrm{n}}$. This width was derived from a simulation of the neutron production inside the $\mathrm{D}_{2}$-filled gas target with the SINENA code [12].

Due to problems with the absolute neutron fluence normalization of the experiment the simulated PHS were scaled on the basis of the ${ }^{12} \mathrm{C}(\mathrm{n}, \alpha){ }^{9} \mathrm{Be}$ peak area. In Fig. 4 a comparison between 
two different normalizations of the simulated PHS is shown for $E_{\mathrm{n}}=16.0 \mathrm{MeV}$. It can be deduced that taking into account not only the peak area but also its low energy tail gives a better agreement between simulation and experiment. This fact suggests that the charge collection properties completely omitted in our simulation - are not yet satisfactorily understood. In Fig. 3 we used the normalization based on the peak area including the low energy tail.

As stated in Section 3 the ranges of charged products of the neutron-induced reactions are included in the simulations. The ranges of $20 \mathrm{MeV}$ protons, deuterons and $\alpha$ particles in carbon amount to $1.3,0.8$ and $0.1 \mathrm{~mm}$ [11], respectively. Therefore the simulations presented here are expected to change significantly only in the extreme cases where the detector size is smaller than or comparable with the aforementioned ranges.

\section{Discussion}

The simulation benchmark suggests that two-body reactions ${ }^{12} \mathrm{C}(\mathrm{n}, \alpha){ }^{9} \mathrm{Be},{ }^{12} \mathrm{C}(\mathrm{n}, \mathrm{p}){ }^{12} \mathrm{~B}$ and ${ }^{12} \mathrm{C}(\mathrm{n}, \mathrm{d}){ }^{11} \mathrm{~B}$ are well described in our simulations. Significant discrepancies are observed in the case of broad distributions which do not describe the measurements well. With a detailed modelling of the individual neutron-induced reactions on an event-by-event basis we achieved a reasonable agreement between the simulated and measured PHS which is qualitatively better than in previous works $[5,6,7]$. However, more reliable differential cross section data are still needed for the $3 \alpha$ breakup channels in ${ }^{12} \mathrm{C}$. In addition, a more detailed simulation including the charge collection properties of the diamond detectors is needed in order to better understand the low energy tail of the ${ }^{12} \mathrm{C}(\mathrm{n}, \alpha){ }^{9}$ Be peak.

\section{References}

[1] A. Zimbal, L. Giacomelli, R. Nolte and H. Schuhmacher, Characterization of monoenergetic neutron reference fields with a high resolution diamond detector, Radiat. Meas. 45, 1313 (2010).

[2] M. Pomorski, E. Berdermann, A. Caragheorgheopol, M. Ciobanu, M. Kiš, A. Martemiyanov, C. Nebel and P. Moritz, Development of single-crystal CVD-diamond detectors for spectroscopy and timing, Phys. Status Solidi A 203, 3152 (2006).

[3] M. Zbořil and A. Zimbal, Response function stability of single crystal diamond detectors to $14 \mathrm{MeV}$ neutrons, Rev. Sci. Instrum. 85, 11D839 (2014).

[4] S.V. Gvozdev, V.V. Frunze and V.N. Amosov, Numerical simulation of the energy spectrum of recoil nuclei and $\alpha$ particles from interactions of fast neutrons with diamond, Instrum. Exp. Tech. 52, 637 (2009).

[5] A. Milocco, A. Trkov and M. Pillon, Simulation of charge collection in diamond detectors irradiated with deuteron-triton neutron sources, AIP Conf. Proc. 1412, 224 (2011).

[6] A. Milocco, M. Pillon, M. Angelone, A. Plompen, A. Krása and A. Trkov, Monte Carlo simulation of the experimental pulse height spectra produced in diamond detectors by quasi-mono-energetic neutrons, Nucl. Instrum. Meth. A 720, 74 (2013).

[7] M. Rebai, A. Milocco, L. Giacomelli, E. Perelli Cippo, M. Tardocchi, A. Fazzi, A. Pietropaolo and G. Gorini, Response of a single-crystal diamond detector to fast neutrons, J. Instrum. 8, P10007 (2013). 
[8] G. Dietze and H. Klein, NRESP4 and NEFF4 - Monte Carlo codes for the calculation of neutron response functions and detection efficiencies for NE 213 scintillation detectors, Report PTB-ND-22, PTB Braunschweig, ISSN 0572-7170 (1982).

[9] F. Gagnon-Moisan, A. Zimbal, R. Nolte, M. Reginatto and H. Schuhmacher, Characterization of single crystal chemical vapor deposition diamond detectors for neutron spectrometry, Rev. Sci. Instrum. 83, $10 \mathrm{D} 906$ (2012).

[10] D. Schmidt, R. Böttger, H. Klein and R. Nolte, Investigation of the ${ }^{9} \mathrm{Be}(\alpha, n){ }^{12} \mathrm{C}$ reaction II: Differential cross sections for $E_{\alpha}=7.02-15.70 \mathrm{MeV}$ and $E_{e x}\left({ }^{12} \mathrm{C}\right)=0.0,4.439,7.654,9.641,10.84$, 11.83 and 12.7 MeV, Report PTB-N-8, PTB Braunschweig, ISBN 3-89429-177-X (1992).

[11] J. Ziegler, Software package SRIM (Stopping and Range of Ions in Matter), www.srim.org (2014).

[12] D. Schmidt and B.R.L. Siebert, Fast neutron spectrometry and Monte Carlo simulation - The codes SINENA and STREUER, Report PTB-N-40, PTB Braunschweig, ISBN 3-89701-531-5 (2000). 